\title{
Authentizität der Wissenschaftsvermittlung im Schülerlabor - Einführung in den Thementeil
}

\author{
Katrin Sommer · Joachim Wirth $\cdot$ Nikol Rummel
}

(C) Springer Fachmedien Wiesbaden GmbH, ein Teil von Springer Nature 2018

\section{Einleitung}

Seit gut 30 Jahren etablieren sich an verschiedenen Einrichtungen wie Universitäten, Museen oder auch Industriestandorten sogenannte „Schülerlabore“ als außerschulische Lernorte. Üblicherweise werden sie von Schulklassen besucht, um sich einen Tag lang außerhalb des gewohnten Schulsettings möglichst eigenständig und unter Nutzung der wissenschaftlichen Methoden der entsprechenden Disziplin mit einem Forschungsthema auseinanderzusetzen. Dabei haben Schülerlabore an Universitäten und Forschungseinrichtungen den Ruf, sie könnten Wissenschaft authentisch oder zumindest authentischer als herkömmlicher Schulunterricht vermitteln. Als Begründung führen Euler et al. (2015, S. 760) an: „Die Labore bieten (...) komplementäre Möglichkeiten zur Anreicherung und Ergänzung des Unterrichts vor allem in Bezug auf authentische, lebensweltbezogene, naturwissenschaftlich-technische Themenfelder und Arbeitsweisen“ (s.a. Guderian 2007; Glowinski 2007). Auch die Schülerlabore selbst sehen die ,selbstständige Auseinandersetzung mit authentischen Forschungs- und Entwicklungszusammenhängen und Arbeitsweisen ..." (S. 5) als eines ihrer grundlegenden Ziele an, wie Dähnhardt et al. (2007) bei einer Analyse der internetbasierten Selbstdarstellungen der Schülerlabore feststellen. Insofern herrscht in der Schülerlabor-Forschung Einigkeit darüber, dass Schülerlabore ,,authentische“ Lernumgebungen darstellen, da sie inhaltlich und methodisch nah an der „echten“ Arbeit von Wissenschaftlerinnen und Wissenschaftlern sind und Lernende dort wie echte Forscherinnen und Forscher handeln können (vgl. auch Haupt und Hempelmann 2015, S. 17).

K. Sommer $(\square) \cdot$ J. Wirth $\cdot$ N. Rummel

Ruhr-Universität Bochum, Universitätsstraße 150, 44801 Bochum, Deutschland

E-Mail: katrin.sommer@rub.de 
Aus einer pädagogisch-psychologischen sowie aus einer fachdidaktischen Perspektive stellt sich jedoch die Frage, ob diese Nähe zur Wissenschaft, ob diese Authentizität bei den Schülerinnen und Schülern, die ein Schülerlabor besuchen, überhaupt etwas bewirkt. Haupt und Hempelmann (2015, S. 17) stellen zwar fest, dass Authentizität „ein entscheidender Faktor für die Wirkung der Schülerlabore“ ist. Diese Feststellung wurde bislang jedoch empirisch noch nicht geprüft. Insofern ist unklar, ob Authentizität tatsächlich ein Merkmal von Schülerlaboren ist, ob dieses Merkmal einen Einfluss auf Lernende hat und wenn ja, welche Effekte bei Lernenden hervorgerufen werden, und wie die Zusammenhangsstruktur zwischen Authentizität des Lernsettings und Lernervariablen aussieht.

An dieser Stelle setzt das 2013 gegründete, interdisziplinär aufgestellte Promotionskolleg „Authentizität der Wissenschaftsvermittlung im Schülerlabor“ der Bochumer Professional School of Education an, aus dessen Kontext die drei Beiträge des Thementeils kommen. Dieses Promotionskolleg nutzt als Ort der Forschung das Alfried Krupp-Schülerlabor, welches durch alle 20 Fakultäten der Ruhr-Universität getragen wird. Dort werden Schülerlaborprojekte aus den Naturwissenschaften (z.B. physikalische Experimente in einem Fallturm zum Thema Schwerelosigkeit) sowie den geistes- und gesellschaftswissenschaftlichen Fächern (z. B. Untersuchungen zum Rassismusbegriff anhand qualitativer sozialwissenschaftlicher Methoden) angeboten. Diese Vielfalt mündet in der Gemeinsamkeit, dass Schülerlaborprojekte Einblicke in die jeweilige Wissenschaft, ihre Objekte und Methoden geben werden.

In mehreren Promotionsprojekten aus verschiedenen Fächern wird untersucht, wie Authentizität der Vermittlung von Wissenschaft zu definieren und zu operationalisieren ist, wie eine solche Authentizität in Schülerlaborprojekten unterschiedlicher Fächer und Disziplinen didaktisch hergestellt werden kann, wie authentisch Schülerinnen und Schüler das wissenschaftliche Denken und Arbeiten in einem solchen Schülerlaborprojekt wahrnehmen und welche Wirkung(en) diese Wahrnehmung hat. Damit sollen die oben skizzierten Fragen der Schülerlabor-Forschung aus pädagogisch-psychologischer wie fachdidaktischer Perspektive in ersten Schritten bearbeitet werden (vgl. Scharfenberg und Bogner 2015).

\section{Authentizität der Wissenschaftsvermittlung}

Wissenschaftsvermittlung zielt nach unserem Verständnis darauf ab, Lernenden das wissenschaftliche Denken sowie die wissenschaftlichen Arbeitsweisen näherzubringen, verständlich zu machen und zu üben. Der Anspruch der Vermittlung von wissenschaftlichen Denk- und Arbeitsweisen an die Lernenden ist immer mit einem Mindestmaß an Didaktisierung des entsprechenden Lernsettings verbunden. Das Ausmaß dieser Didaktisierung ist wiederum entscheidend für das Ausmaß an Authentizität der Wissenschaftsvermittlung. Je stärker ein Lernsetting durch instruktionale und didaktische Entscheidungen Lehrender geprägt ist, desto stärker unterscheidet es sich von einem Setting, in dem Wissenschaftlerinnen und Wissenschaftler in Realität Wissenschaft betreiben und desto geringer ist seine Authentizität ausgeprägt. Dagegen steigert sich die Authentizität der Wissenschaftsvermittlung in dem Maße, wie das Lernsetting sich an die verschiedenen Facetten der realen wissenschaftlichen 
Forschungs- und Berufswelt annähert (Pawek 2009). Insofern lässt sich Authentizität als Ausprägung auf einer Dimension verstehen, die sich zwischen Realität (der wissenschaftlichen Forschungs- und Berufswelt) und vollständig didaktisiertem Lernsetting aufspannt. Dabei gilt jedoch, dass die Authentizität des Lernsettings an beiden Polen der Dimension verloren geht. Auf der einen Seite hat ein vollständig didaktisiertes Lernsetting mit der realen wissenschaftlichen Forschungs- und Berufswelt nichts mehr zu tun. Auf der anderen Seite umfasst die reale Forschungs- und Berufswelt weit mehr als in einem Lernsetting abbildbar wäre (man denke bspw. an die Arbeiten an Anträgen oder in universitären Gremien, die zur Berufswelt von Wissenschaftlerinnen und Wissenschaftlern gehören, aber nicht Teil eines Schülerlaborprojekts sind). Würde man Schülerinnen und Schüler nicht in ein authentisches Schülerlabor, sondern in diese reale Forschungs- und Berufswelt schicken, müssten sie nicht authentisch wie, sondern real als Forscherinnen und Forscher handeln, was keiner Schülerin und keinem Schüler zuzumuten ist. Insofern unterscheidet sich jedes Lernsetting, selbst die authentischsten Schülerlaborprojekte, immer und zurecht von der Realität.

Ist man daran interessiert, Lernsettings wie z. B. Schülerlaborprojekte möglichst authentisch zum Zweck der Wissenschaftsvermittlung zu gestalten, ergibt sich die Frage, welche der von Pawek (2009) angesprochenen Facetten der realen wissenschaftlichen Forschungs- und Berufswelt zu beachten sind. Basierend auf Ansätzen u. a. von Chinn und Malhorta (2002), Glowinski (2007) oder Hsu et al. (2010) schlagen Betz et al. (2016) sieben Merkmale von Lernsettings vor, die durch Lehrende gestaltet werden können und die das Ausmaß an Authentizität des Lernsettings beeinflussen (Abb. 1). Hierzu zählen sie den Ort, das Material, die Methoden, den Inhalt, die Innovation, das soziale Setting sowie den Vermittler bzw. die Vermittlerin. Der Ort umfasst Gebäude, Räume und Orte, in denen die Wissenschaftsvermittlung stattfindet. Diese sind authentisch, wenn sie für wissenschaftliche Zwecke errichtet, eingerichtet oder hergerichtet wurden, so dass in ihnen Wissenschaft betrieben wurde, wird oder zumindest werden kann. Zum Material zählen alle Dinge, die sowohl Wissenschaftlerinnen und Wissenschaftler als auch Lernende entweder untersuchen (z. B. Bodenproben oder Zeitzeugeninterviews) oder aber nutzen, um etwas zu untersuchen (z. B. Geräte oder Instrumente). Unter Methoden werden sowohl die prinzipiellen Vorgehensweisen (im Sinne wissenschaftlicher Denk- und Arbeitsweisen) als auch die konkrete Nutzung von Geräten und Instrumenten verstanden. Sie sind authentisch, wenn sie dem wissenschaftlichen Vorgehen der jeweiligen Disziplin entsprechen und einen Prozess der systematischen Erkenntnisgewinnung gestalten. Der Inhalt entspricht dem Thema bzw. der untersuchten Fragestellung. Er ist authentisch, wenn er einem Forschungsthema der jeweiligen Disziplin entspricht. Innovativ ist ein Thema bzw. eine Fragestellung dann, wenn durch ihre Beantwortung ein tatsächlicher Erkenntnisgewinn für die jeweilige Disziplin (und nicht nur für die Lernenden selbst) entsteht und das Agieren der Lernenden nicht aus einer reinen Rezeption oder Replikation wissenschaftlicher Kenntnisse besteht. Das soziale Setting umfasst alle Personen, mit denen Lernende interagieren, wozu auch die lehrende Person als Wissenschaftsvermittlerin bzw. -vermittler gehört. Diese Personen sind authentisch, wenn sie in ihrem beruflichen Alltag als Wissenschaftlerinnen 


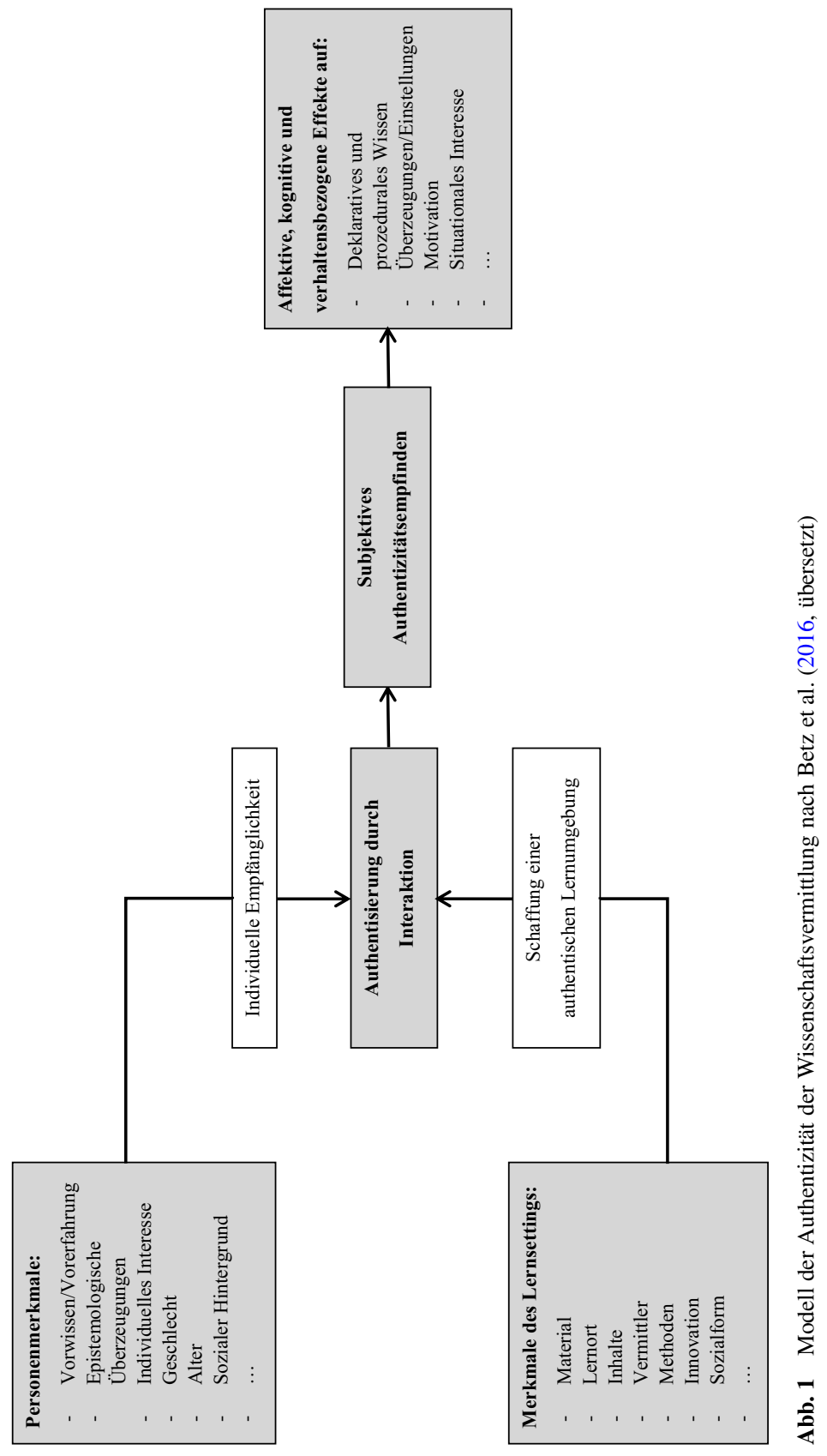


oder Wissenschaftler handeln und den Zugang für Lernende zu wissenschaftlicher Forschung der jeweiligen Disziplin herstellen und gewährleisten.

Betz et al. (2016) verstehen die vorgeschlagenen sieben Merkmale nicht als erschöpfende Liste von Merkmalen, die die Authentizität eines Lernsettings bestimmen. Sie sind jedoch über wissenschaftliche Disziplinen hinweg begründbar und könnten in Summe das Ausmaß an Authentizität eines Lernsettings maßgeblich beeinflussen. Zudem sind es Merkmale, die insbesondere in Schülerlaboren für eine authentische Wissenschaftsvermittlung realisiert werden können. Ihre empirische Überprüfung steht bislang zwar noch aus. Erste Schritte unternehmen jedoch Betz (2018), Mierwald et al. (2018) und Nachtigall et al. (2018) in diesem Themenheft.

\section{Wahrnehmung von Authentizität der Wissenschaftsvermittlung}

Die verschiedenen Merkmale eines Lernsettings können unterschiedlich authentisch ausgeprägt sein und in ihrer Summe die Authentizität des Lernsettings bestimmen (Betz et al. 2016). Diese „objektive“ Authentizität des Lernsettings kann jedoch für sich allein genommen keine Wirkung im Sinne von Haupt und Hempelmann (2015) erzeugen. Betz et al. (2016) nehmen in ihrem Modell an, dass diese Situationsmerkmale des Lernsettings mit Merkmalen der Lernenden interagieren und Lernende durch diese Interaktion ein Authentizitätsempfinden entwickeln und Authentizität wahrnehmen (van Lier 1996). Letztlich ist es diese Authentizitätswahrnehmung (und nicht die ,objektive“ Authentizität des Lernsettings), die verschiedene affektive, kognitive oder verhaltensbezogene Effekte auf Merkmale von Lernenden hervorrufen kann (Abb. 1).

In ihrem Modell führen Betz et al. (2016) verschiedene Merkmale von Lernenden an, die potenziell in Interaktion mit den situativen Merkmalen des Lernsettings die Authentizitätswahrnehmung Lernender beeinflussen. Dazu zählen instruktional und didaktisch beeinflussbare Merkmale wie Vorerfahrungen und Vorwissen, epistemologische Überzeugungen oder Interesse, aber auch stabile oder durch Lehrende kaum beeinflussbare Merkmale wie Geschlecht, Alter oder sozialer Hintergrund der Lernenden. Eine Kombination der verschiedenen Charakteristika der Lernenden macht nach dem Modell Lernende mehr oder weniger empfänglich für Authentizität und beeinflusst so die Wahrscheinlichkeit, dass Lernende ein Lernsetting authentisch wahrnehmen.

\section{Authentizität der Wissenschaftsvermittlung als Forschungsgegenstand}

Das Modell von Betz et al. (2016) ist ein Ansatz, den etwas schillernden Begriff der Authentizität mit Blick auf Wissenschaftsvermittlung theoretisch klarer zu fassen und einen theoretischen Rahmen als Ausgangspunkt für empirische Studien zu bieten. Damit ist ein erster Schritt getan, allerdings eben nur ein erster. Das Modell bzw. die sich daraus ableitbaren Merkmale authentischer Lernsettings und ihre Bedeutung für die Entwicklung verschiedener Merkmale der Lernenden wie Wissen, Interesse, 
epistemologische Überzeugungen, sind bislang empirisch noch nicht überprüft. Hier besteht noch ein substanzieller Forschungsbedarf.

Schülerlabore, wie z. B. das Alfried Krupp-Schülerlabor der Ruhr-Universität Bochum mit seinem naturwissenschaftlichen und seinem geistes- und gesellschaftlichen Bereich, bieten für eine solche Forschung ein hervorragendes Umfeld. Schülerlabore sind nicht nur Lern-Labore für Schülerinnen und Schüler, sondern können auch als Forschungs-Labore dienen (Schwarzer und Parchmann 2018), in denen pädagogisch-psychologische und fachdidaktische Forschung stattfindet (z. B. Itzek-Greulich et al. 2015; Scharfenberg und Bogner 2010; Sommer et al. 2013). Die laborartigen Bedingungen ermöglichen auch, dass beispielsweise einzelne Merkmale eines Lernsettings systematisch zu variieren und die entsprechenden affektiven, kognitiven oder verhaltensbezogenen Effekte auf Schülerinnen und Schüler unter kontrollierten Bedingungen zu untersuchen.

In diesem Schülerlaborkontext sind die empirischen Arbeiten entstanden, die in diesem Thementeil vorgestellt werden. Alle drei haben gemeinsam, dass sie für die empirische Bearbeitung ihrer Forschungsfragen das Alfried Krupp-Schülerlabor genutzt haben. Alle drei haben dabei einen experimentellen Ansatz gewählt, in dem sie einzelne Merkmale des Lernsettings nach Betz et al. (2016) variierten und verschiedene affektive sowie kognitive Effekte dieser Variation untersuchten. Dabei gehen alle drei Arbeiten davon aus, dass diese Effekte nicht direkt, sondern über die Authentizitätswahrnehmung vermittelt werden (auch wenn nicht alle Arbeiten diese Annahme empirisch prüfen).

Etwas untypisch für die Schülerlaborforschung sind die durch die drei Beiträge abgedeckten Fächer, weil sie nicht wie sonst üblich aus den Naturwissenschaften kommen. Betz (2018) arbeitet mit einem germanistischen Schülerlaborprojekt, Mierwald et al. (2018) stellt eine Studie aus der Geschichtsdidaktik vor. Nachtigall et al. (2018) untersuchen ein erziehungs- und sozialwissenschaftliches Schülerlaborprojekt und tragen aus der Pädagogischen Psychologie zum Thementeil bei.

Abgesehen von den Fächern unterscheiden sich die drei Arbeiten sowohl in Bezug auf die experimentell manipulierten Merkmale des Lernsettings als auch in Bezug auf die untersuchten Effekte. Betz (2018) nimmt als Merkmale des Lernsettings den Ort und den Vermittler in den Blick und argumentiert, dass im Kontext eines Schülerlabors einer Hochschule diese beiden Merkmale untrennbar voneinander die Authentizität der Lernumgebung maßgeblich bestimmen. Zudem kann sie in ihrer experimentellen Interventionsstudie zeigen, dass diese Authentizität der Lernumgebung einen Einfluss auf das situationale Interesse der Schülerinnen und Schüler hat, und dass dieser Einfluss über die Authentizitätswahrnehmung mediiert wird. Bei Mierwald et al. (2018) steht das Material im Vordergrund. Sie untersuchen, inwiefern die Authentizität des Materials, mit dem Schülerinnen und Schüler in einem Schülerlaborprojekt $\mathrm{zu}$ einem geschichtlichen Thema arbeiten, ihre epistemologischen Überzeugungen beeinflusst. Über eine mehr oder weniger starke didaktische Aufbereitung historischer Quellen und Darstellungen variieren sie deren Wissenschaftsnähe und damit deren Authentizität (im Kontext von Wissenschaftsvermittlung). Die Ergebnisse zeigen, dass eine hohe Authentizität des Materials eine kritisch-reflektierte epistemologische Überzeugungshaltung begünstigt, während stark didaktisiertes Material, beispielsweise in Form von Schulbuchtexten, keine (kurzfristige) Verän- 
derung der epistemologischen Überzeugungen hervorbringt. Nachtigall et al. (2018) fokussieren die Methode bzw. die Vorgehensweise der Schülerinnen und Schüler bei der Bearbeitung einer Problemstellung als Merkmal des Lernsettings, finden in ihrer quasi-experimentellen Untersuchung jedoch keine Effekte der variierten Authentizität der Methode auf die Authentizitätswahrnehmung oder das situationale Interesse von Schülerinnen und Schülern.

Die in diesem Thementeil zusammengetragenen Beiträge sind erste empirische Bausteine zur Untersuchung der Bedeutung von Authentizität der Wissenschaftsvermittlung für eine kleine Auswahl von affektiven und kognitiven Faktoren wie Wissen, Interesse oder epistemologische Überzeugungen. Alle drei fokussieren Merkmale des Lernsettings. In Ergänzung dazu untersuchte im Rahmen des Promotionskollegs Vanderbeke (2017) Authentisierungsprozesse zwischen Schülerinnen und Schülern und der Lernumgebung eines molekular-biologischen, bilingualen Schülerlabors. Mit einem qualitativen Ansatz fand sie Hinweise auf die große Bedeutung von Vorwissen und Vorerfahrungen, aber auch von stereotypen, medial geprägten Vorstellungen von Wissenschaftlichkeit, die die Authentisierungsprozesse sowie die Möglichkeit der Schülerinnen und Schüler, die ihnen angebotene Rolle eines Wissenschaftlers anzunehmen, beeinflussen. Sie zeigte, dass Merkmale des Lernsettings, die von den Urhebern eines Schülerlaborprojektes als authentizitätssteigernd angenommen wurden (in diesem Fall die Kommunikation auf Englisch statt auf Deutsch), auch befremdlich (statt wissenschaftsnah) und kommunikationshemmend wirken und damit ebenfalls die Identifikation mit der Wissenschaftlerrolle erschweren können.

Zusammengenommen zeigen die Studien zweierlei. Zum einen wird deutlich, dass die Frage nach der Bedeutung der Authentizität der Wissenschaftsvermittlung in Lehr-Lernkontexten theoretisch wie empirisch noch lange nicht hinreichend beantwortet ist. Das von Betz et al. (2016) vorgeschlagene Modell ist ein erster wertvoller theoretischer Ansatz, der jedoch sicherlich an der einen oder anderen Stelle noch überarbeitet werden muss. So zeigt die Arbeit von Vanderbeke (2017), dass die Bedeutung des Vorwissens und der Vorerfahrungen von Schülerinnen und Schülern in dem Modell noch nicht hinreichend berücksichtigt ist. Darüber hinaus ist die Erfassung der Authentizität der Wissenschaftsvermittlung ein noch nicht befriedigend gelöstes Problem. Zum anderen zeigen die Studien in diesem Thementeil jedoch auch, dass es sich lohnt, dieser Frage wissenschaftlich-empirisch nachzugehen. Die ersten empirischen Ergebnisse liefern wertvolle Hinweise für die Gestaltung von Lernsettings zur authentischen Vermittlung wissenschaftlicher Denk- und Arbeitsweisen, nicht nur im Rahmen von Schülerlaboren. Sie haben das Potenzial dazu beizutragen, Schülerinnen und Schüler für das wissenschaftliche Arbeiten in den verschiedenen Fächern und Disziplinen zu begeistern. Damit erhalten diese und weitere Ergebnisse in dem Forschungsfeld nicht nur wissenschaftliche, sondern auch bildungspolitische Relevanz.

Danksagung Wir danken Nicola Brauch, Henning Feldmann, Wolfgang Kirchner, Christiane Mattiesson, Claudia Müller-Brauers, Irene Neumann, Helmut Pulte, Björn Rothstein, Sandra Sprenger, Julia Suckut und Eva Wilden für die konstruktive Zusammenarbeit im Promotionskolleg. 
Ein weiterer Dank gilt dem Ministerium für Kultur und Wissenschaft des Landes Nordrhein-Westfalens für die finanzielle Unterstützung im Rahmen der Förderlinie „Ausbau der Fachdidaktik“. Diese Mittel haben der Professional School of Education der Ruhr-Universität Bochum die Gründung des Promotionskollegs erst ermöglicht, in dessen Rahmen alle empirischen Beiträge dieses Thementeils entstanden.

\section{Literatur}

Betz, A. (2018). Der Einfluss der Lernumgebung auf die (wahrgenommene) Authentizität der linguistischen Wissenschaftsvermittlung und das Situationale Interesse von Lernenden. Erscheint in diesem Thementeil der Unterrichtswissenschaft.

Betz, A., Flake, S., Mierwald, M., \& Vanderbeke, M. (2016). Modelling authenticity in teaching and learning contexts: a contribution to theory development and empirical investigation of the construct. In C. K. Looi, J. Polman, U. Cress \& P. Reimann (Hrsg.), Transforming learning, empowering learners: the International Conference of the Learning Sciences (ICLS) 2016 Vol. 2 (S. 815-818). Singapur: International Society of the Learning Sciences.

Chinn, C. A., \& Malhotra, B. A. (2002). Epistemologically authentic inquiry in schools: a theoretical framework for evaluating inquiry tasks. Science Education, 86, 175-218.

Dähnhardt, D., Sommer, K., \& Euler, M. (2007). Lust auf Naturwissenschaft und Technik - Lernen im Schülerlabor. Unterricht Chemie, 18(99), 4-9.

Euler, M., Schüttler, T., \& Hausamann, D. (2015). Schülerlabore: Lernen durch Forschen und Entwickeln. In E. Kircher \& al (Hrsg.), Physikdidaktik (S. 759-782). Berlin: Springer.

Glowinski, I. (2007). Schülerlabore im Themenbereich Molekularbiologie als Interesse fördernde Lernumgebungen. Dissertationsschrift, Christian-Albrechts-Universität zu Kiel.

Guderian, P. (2007). Wirksamkeitsanalyse außerschulischer Lernorte - Der Einfluss mehrmaliger Besuche eines Schülerlabors auf die Entwicklung des Interesses an Physik. Dissertationsschrift, HumboldtUniversität zu Berlin.

Haupt, O., \& Hempelmann, R. (2015). Schülerlabore in Art und Form. Eine Typensache! In O. J. Haupt, et al. (Hrsg.), Schülerlabor-Atlas 2015. Schülerlabore im deutschsprachigen Raum (S. 14-21).

Hsu, P.-L., van Eijck, M., \& Roth, W.-M. (2010). Student's representations of scientific practice during science internship: reflections from activity-theoretic perspective. International Journal of Science Education, 32, 1243-1266.

Itzek-Greulich, H., Flunger, B., Vollmer, C., Nagengast, B., Rehm, M., \& Trautwein, U. (2015). Effects of a science center outreach lab on school students' achievement - Are student lab visits needed when they teach what students can learn at school? Learning and Instruction, 38, 43-52.

Van Lier, L. (1996). Interaction in the language curriculum: awareness, autonomy and authenticity. London, New York: Longman.

Mierwald, M., Lehmann, T. \& Brauch, N. (2018). Zur Veränderung epistemologischer Überzeugungen im Schülerlabor: Authentizität von Lernmaterial als Chance der Entwicklung einer wissenschaftlich angemessenen Überzeugungshaltung im Fach Geschichte? Erscheint in diesem Thementeil der Unterrichtswissenschaft.

Nachtigall, V., Rummel, N. \& Serova, K. (2018). Authentisch ist nicht gleich authentisch - Wie Schülerinnen und Schüler die Authentizität von Lernaktivitäten im Schülerlabor einschätzen. Erscheint in diesem Thementeil der Unterrichtswissenschaft.

Pawek, Chr. (2009). Schülerlabore als interessefördernde außerschulische Lernumgebungen für Schülerinnen und Schüler aus der Mittel- und Oberstufe. Dissertationsschrift, Christian-Albrechts-Universität zu Kiel.

Scharfenberg, F.-J., \& Bogner, F.X. (2015). Empirische Analyse: Leistung zählt! In O. J. Haupt, et al. (Hrsg.), Schülerlabor-Atlas 2015. Schülerlabore im deutschsprachigen Raum (S. 24-31). Stuttgart: Klett MINT.

Scharfenberg, F. J., \& Bogner, F. X. (2010). Instructional efficiency of changig cognitive load in an out-ofschool laboratory. International Journal of Science Education, 32(6), 829-844.

Schwarzer, S., \& Parchmann, I. (2018). Außerschulische Lernorte. In K. Sommer, J. Wambach-Laicher \& P. Pfeifer (Hrsg.), Konkrete Fachdidaktik Chemie (S. 668-680). Seelze: Friedrich-Verlag.

Sommer, K., Russek, A., Kleinhorst, H., Kakoschke, A., \& Efing, N. (Hrsg.) (2013). KEMIE - Kinder erleben mit ihren Eltern Chemie. Chemkon, 20(5), 209-352.

Vanderbeke, Marie (2017). Authentisierungsprozesse und die Nutzung fremdsprachlicher Affordances in bilingualen Schülerlaborprojekten. Dissertationsschrift, Ruhr-Universität Bochum. 